\title{
An STDMA-Based Framework for QoS Provisioning in Wireless Mesh Networks
}

\author{
Mauro Leoncini, Paolo Santi, Paolo Valente
}

\begin{abstract}
Providing strong QoS guarantees for wireless multihop networks is very challenging, due to many factors such as use of a shared communication medium, variability in wireless link quality, and so on. However, wireless mesh technology gives the opportunity to alleviate some of these problems, due to lack of mobility in the wireless infrastructure, and presence of natural centralization points in the network. The main contribution of this paper is the definition of a simple framework that exploits these features to provide provable, strong QoS guarantees to network clients. In particular, admitted clients are guaranteed a certain minimum bandwidth and maximum delay on their connections. The framework is based on Spatial-TDMA scheduling at the MAC layer, which is periodically executed at the network manager to adapt to changes in traffic demand. Using STDMA has two potential advantages, namely $i$ ) aggregate throughput improvements with respect to CSMA-based solutions; and $i i$ ) careful characterization of each wireless link's performance. While scheduling computation is centralized, admission control is performed locally at the wireless backbone nodes, thus reducing signaling. We propose two bandwidth distribution and related admission control policies, which are at opposite ends of the network utilization/spatial fairness tradeoff. Through extensive simulations, we show that the proposed framework achieves its design goals of providing strong QoS guarantees to (VoIP clients) while not sacrificing throughput in a realistic mesh network scenario, also in presence of highly unbalanced load at the backbone nodes. To the best of our knowledge, this is the first proposal with similar features for wireless mesh networks.
\end{abstract}

Index Terms-wireless multi-hop networks, spatial TDMA, Quality of Service, physical interference model.

\section{INTRODUCTION}

Wireless mesh networks are a very promising technology for providing ubiquitous broadband wireless access, mainly due to their ease of deployment and maintenance. However, several challenges are still to be faced for these premises to be fulfilled. The main challenges are related to an efficient use of the wireless spectrum to reduce interference and increase capacity, and to provide QoS guarantees to the network clients.

Providing QoS guarantees to clients of a wireless multi-hop network is a very challenging, long standing problem. Here, difficulties lie in the fact that a very basic component of any QoS provisioning framework (i.e., accurate characterization of link-level performance) is hard to achieve, due to factors such as use of shared communication medium, variability in radio channel conditions, possible node mobility, and so on.

A preliminary, shorter version of this paper appeared in Proc. IEEE Int. Conference on Mobile Ad Hoc and Sensor Systems (MASS), 2008.

M. Leoncini and P. Valente are with the Dip. Ing. dell'Informazione, Univ. of Modena e Reggio Emilia, ITALY.

Paolo Santi is with IIT-CNR, Pisa, ITALY. The work of P. Santi has been partially supported by IIT-CNR under Project P056: "Networks for Critical Infrastructures and Web Analysis".
However, mesh networks have peculiar features with respect to other types of wireless multi-hop networks which may help a lot in the definition of such a QoS framework. In particular, mesh networks are characterized by the presence of a wireless infrastructure, and of natural centralization points (e.g., gateway nodes).

The main contribution of this paper is showing how to exploit these features (lack of mobility in the wireless infrastructure, and presence of centralization points) to build a relatively simple framework able to provide strong (i.e., worstcase) QoS guarantees to network clients. This is in sharp contrast with similar frameworks proposed for other types of wireless multi-hop networks, which typically only achieve weak (i.e., statistical) QoS guarantees.

Our framework is based on exploiting Spatial-TDMA scheduling [30] at the MAC layer. Differently from TDMA, STDMA allows simultaneous transmissions in a transmission slot, as long as these transmissions are sufficiently separated in the space (spatial reuse). If spatial reuse is carefully controlled, several simultaneous transmissions can take place in a slot without corrupting each other, with a considerable potential throughput benefit.

Using STDMA-based MAC is deemed feasible in a wireless mesh network, due to the quasi-static topology of the wireless infrastructure, and the presence of centralization points. Indeed, several recent papers are concerned with applying STDMA-based approaches in wireless mesh networks [3], [11], [17], [32], and forthcoming standards for wireless mesh networks such as 802.16 and 802.11s consider TDMA scheduling as a possible MAC layer mechanism. Concerning presence of centralization points, we observe that the concept of managed mesh network is becoming very popular, e.g., in enterprises and campuses, where a single authority deploys and manages the entire network, deployment is planned, and a centralized service is often available.

Exploiting STDMA at the MAC layer allows mitigating unpredictability of wireless link performance due to the shared nature of the communication medium, since transmissions are carefully scheduled in such a way that they do not conflict with each other. Hence, up to changes in radio channel properties (which are not addressed in this work), the performance of each network link becomes in principle predictable in a very accurate way. Clearly, accuracy of link performance predictions heavily depends on the interference model used to build the transmission schedule. Recent research based on testbed experiments has shown that SINR-based interference models, according to which correct message reception depends on the Signal-to-Interference-and-Noise-Ratio experienced at the receiver, provide much better accuracy (and, consequently, 
throughput benefits) with respect to simpler interference models [27], [28]. In this paper, we consider an instance of such SINR-based interference models (namely, the physical interference model of [16]) to build the transmission schedule. Hence, we expect our framework has the potential to provide relatively accurate link quality performance predictions in a real application scenario.

The key of our framework, which we call WoW (Wired over Wireless), is to use such accurate link performance estimation to provide strong QoS guarantees to the wireless backbone nodes composing the wireless mesh network infrastructure, which can be turned into strong QoS guarantees provided to the end user if proper scheduling and admission control policies are locally executed at the backbone nodes.

In this paper, we formally prove that the WoW framework, when complemented with simple backbone node-level policies, provides strong QoS guarantees to the end user in terms of minimum available bandwidth and maximum possible delay on her connection to a gateway node. Furthermore, we report the results of extensive packet-level simulation experiments that confirm the theoretical analysis in a realistic VoIP application scenario. The simulation results also show WoW's ability to provide strong QoS guarantees to VoIP users and to effectively use network bandwidth even in presence of highly unbalanced network load. To the best of our knowledge, WoW is the first framework for QoS provisioning in mesh networks with similar features proposed in the literature.

\section{RELATED WORK AND CONTRIBUTION}

The issue of providing QoS guarantees in wireless multihop networks has been widely investigated in the literature, in particular with reference to wireless ad hoc networks. QoS constraints have been considered both at the MAC layer [29], [34], [36], [38], at the routing layer [24], [25], [26], [35], and in cross-layer solutions [14], [22]. For a survey on QoS approaches for wireless ad hoc networks, the reader is referred to [33]. Given the fully distributed, mobile nature of ad hoc networks, accurately characterizing bandwidth and delay characteristics of each link in the network (which is the basic ingredient for providing strong QoS guarantees) is very challenging. This is the reason why typical QoS approaches for ad hoc networks can provide only weak (e.g., statistical) guarantees on QoS level guaranteed to clients.

A few approaches specifically targeted to mesh networks have recently been introduced in the literature. In [4], [10], the problem of optimal selection of gateway nodes in order to provide QoS guarantees to clients is considered. A major limitation of these approaches is that only primary interference is considered when determining QoS guarantees. This means that the only constraint when determining feasibility of a transmission set is that a node cannot be at the endpoint of two or more active links. In [20], the authors are concerned with dimensioning a wireless mesh network in such a way that certain flow-level QoS requirements are fulfilled. Similarly to [4], [10], the approach of [20] is based on a TDMA MAC layer, and uses a simplified interference model.

The authors of [9] are concerned with fairness, and presents a max-min fairness approach based on TDMA scheduling.
Fairness is also the main focus of [15], in which the authors show that nodes which are a few hops away from the gateway are severely penalized in 802.11-based mesh networks.

In [18], the authors address the QoS issue in wireless mesh networks at the routing level. The authors propose a technique for accurately estimating end-to-end delay in an 802.11-based mesh networks. The proposed QoS-aware routing protocol addresses also the problem of route stability, privileging relatively stable routes over relatively unstable ones. Although much more accurate end-to-end delay estimation is achieved with the proposed technique, a significant control overhead is introduced, with negative effects on network throughput.

Perhaps the works more related to ours are [13], [37]. In both these works, the authors have the similar goal of providing QoS guarantees (in terms of bandwidth and delay constraints) to the final users. However, [37] is based on 802.11 MAC, and on simplistic interference models based on the notion of conflict graph. Given this, accuracy of predicted bandwidth/delay estimation provided by the framework proposed in [37] becomes an issue. On the other hand, the approach of [13], although based on TDMA at the MAC layer, completely neglects the effect of radio interference, since it assumes that enough radio resources are available at the backbone nodes so that an arbitrary number of simultaneous transmissions can occur without mutual interference (e.g., enough orthogonal communication channels are available for communication). Unfortunately, this assumption is hardly met in a practical system, where the amount of available radio resources is severely constrained (e.g., only a few orthogonal channels available in $802.11 \mathrm{a} / \mathrm{b} / \mathrm{g}$ ).

To the best of our knowledge, ours is the first QoS approach for wireless mesh networks based on STDMA, coupled with a very accurate interference model, namely the physical interference model introduced in [16]. The difficulty in using this interference model for transmission scheduling lies in the fact that interference generated even by far-away nodes is accounted for when determining whether a set of concurrent transmission is feasible. Only recently a computationally feasible approach to build an STDMA schedule based on the physical interference model has been proposed in the literature [11]. The scheduling algorithm presented in [11], called GreedyPhysical, exhibits low running time when executed on networks of even moderate size (a hundred of nodes), and it is shown to achieve up to three-fold performance improvements in terms of throughput with respect to 802.11based networks. As testbed experimental results reported in [27], [28] confirm, usage of an accurate interference model allows feasible transmission sets to be accurately computed in the schedule, resulting in high link reliability and aggregate throughput improvements. On the other hand, packet reception cannot be ensured with $100 \%$ probability, due to the wellknown time-varying behavior of radio signal. This paper is not concerned with techniques for concealing this type of link failures. Hence, to ease presentation in the following we assume that this type of (unavoidable) failures are dealt with standard techniques, as, e.g., packet retransmission or Forward Error Correction [12] at some higher level than MAC. Thus, when we mention "strong" QoS-guarantees, we mean 
guarantees up to the above mentioned, seldom occurring, packet losses.

In this paper, we propose to use GreedyPhysical as a building block of a simple, centralized STDMA framework for providing strong, end-to-end guarantees in a wireless mesh network. End-to-end guarantees are on both minimum bandwidth and maximum delay, and are strong in that they are guaranteed in the worst-case. The framework is based on a very accurate estimation of minimum bandwidth and maximum delay on each network link, which renders the performance of the network in principle predictable as in a wired network. This is the reason why we have termed our framework WoW (Wired over Wireless). Accurate bandwidth/delay estimation of network links is made available by the use of very accurate interference modeling when building the STDMA schedule.

\section{NETWORK MODEL}

We consider a wireless mesh network composed of $n$ nodes (wireless routers), some of which have direct, wired connection to the Internet and act as gateway nodes. For the ease of reading, in the rest of this paper we will refer to gateway nodes simply as gateways, while we reserve the term node as a shorthand for "non-gateway backbone node". All possible links between nodes (and gateways) are represented by the communication graph $G=(V, E)$, where $e=(u, v) \in E$ if and only if there exists a communication link (in the absence of interference) between $u$ and $v$, where $u, v$ are either nodes or gateways. We restrict our attention to bi-directional links only, i.e., we do not consider, for routing packets, possible uni-directional links in the communication graph.

We assume that packets to/from nodes from/to gateways are routed along shortest path trees rooted at the gateways. In other words, for each node $u$, we suppose that $u$ routes its packets toward a closest gateway $v$, where distance is simply measured as hop count, and with ties broken by means of random choices. However, we require that the routing protocol guarantees that the final routing graph is a forest, with every tree rooted at a gateway. This can be easily done by augmenting $G$ with a dummy node $S$ linked to (and only to) the gateways, and then computing a shortest path tree rooted at $S$. Note that our working assumption of routing along disjoint trees is realistic in a wireless mesh scenario.

The WoW framework is aimed at providing node-level QoS guarantees, and it is independent of the policies implemented within the nodes. However, to assess WoW effectiveness in providing QoS guarantees to final users, we assume that (mobile) wireless clients are present, which issue connection requests to a node within their radio range. Hence each node ${ }^{1}$ $u$ is associated with a dynamic set of clients, which we shall denote by $C(u)$. We assume clients are QoS sensitive. In particular, a generic client $a$ is characterized by two QoS parameters, namely a minimum bandwidth requirement, $b w(a)$, and a maximum delay constraint $\operatorname{del}(a)$, which we will together refer to as the QoS demand of $a$ : $\operatorname{QoS}(a)=$

\footnotetext{
${ }^{1}$ For simplicity, we assume that clients cannot be directly associated with gateways.
}

$\{b w(a), \operatorname{del}(a)\}$. For each node $u$, the value $\sum_{a \in C(u)} b w(a)$ will be taken as $u$ 's bandwidth requirement.

As it is typically the case in wireless mesh networks, we assume that client-to-infrastructure (C2I) and infrastructure-toinfrastructure (I2I) communications use disjoint set of wireless resources, i.e., that C2I and I2I communications do not interfere with each other. This is made possible, for instance, by usage of at least two radios on the wireless routers, and, say, usage of different technologies (e.g., 802.11a and $802.11 \mathrm{~b} / \mathrm{g}$ ) for C2I and I2I communications ${ }^{2}$.

\section{THE WOW FRAMEWORK}

A system view of the WoW framework is depicted in Figure 1. Four types of actors are considered: a network manager, gateways, nodes, and clients.

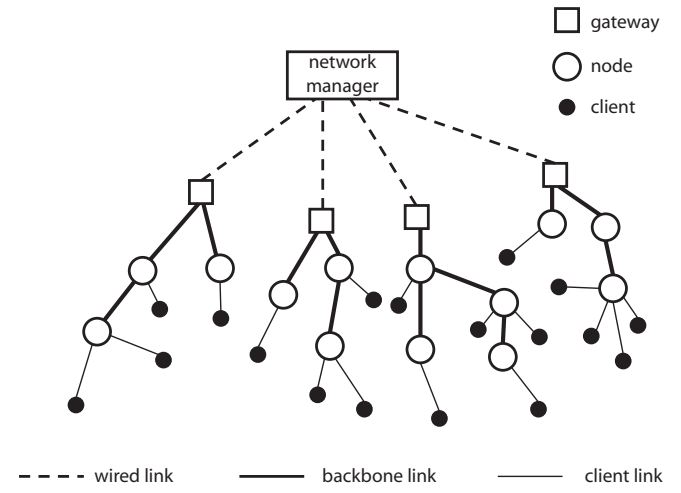

Fig. 1. The WoW framework.

The network manager, which may possibly coincide with one of the gateways, periodically computes the transmission schedule based on node bandwidth requirements ${ }^{3}$. Then, it broadcasts to each gateway $g$ the schedule and the bandwidth allocated to each node in the tree rooted at $g$.

In turn, each gateway takes care of delivering the transmission schedule and the bandwidth allocation to each node in its tree. Also, in the opposite direction, it collects bandwidth requirements from nodes, and delivers them to the network manager.

Nodes realize the important task of converting the aggregate QoS guarantees provided by the WoW framework into clientlevel guarantees.

Finally, as already pointed out, there are wireless clients, which issue connection requests to a node within their radio range. In case of successful request, they are allowed to send/receive packets to/from a gateway node.

\section{A. MAC level scheduling}

The WoW framework is based on the existence of an STDMA protocol at the MAC layer, according to which packet

\footnotetext{
${ }^{2}$ An alternative is to use disjoint set of orthogonal frequency channels for $\mathrm{C} 2 \mathrm{I}$ and I2I communications.

${ }^{3}$ Given the much higher bandwidth and lower delay of a wired link as compared to a wireless link, we assume that message exchange between gateways and the network manager occurs with a negligible delay.
} 


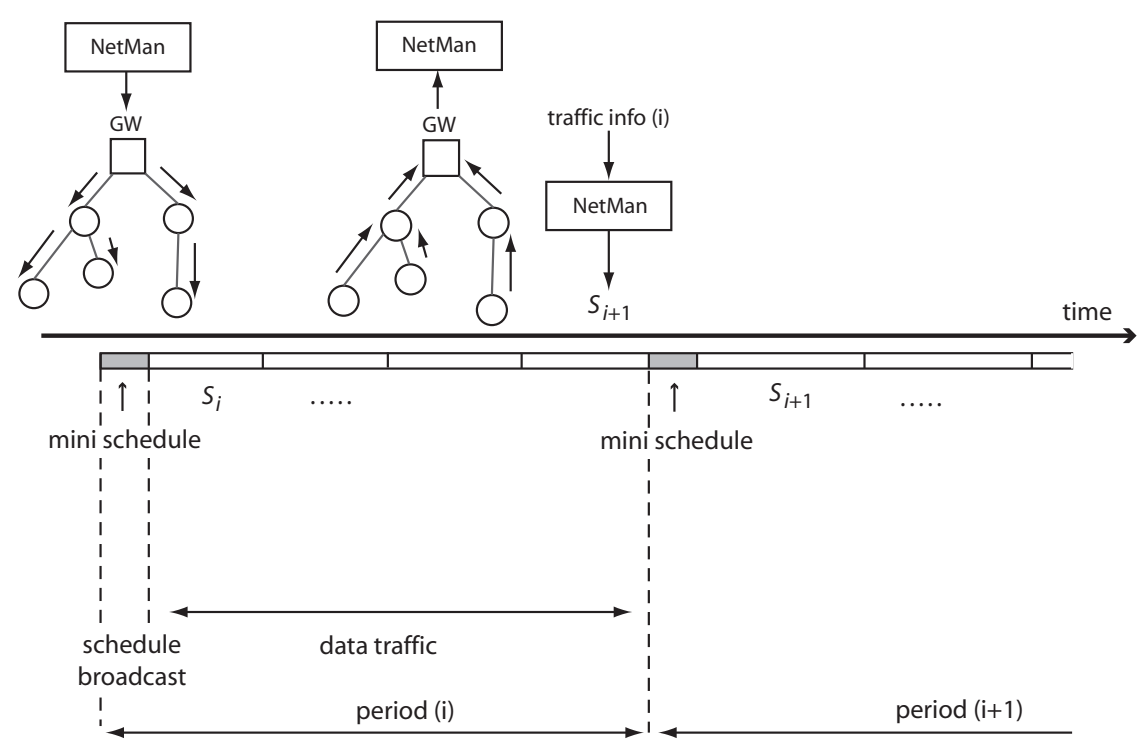

Fig. 2. Adaptive schedule computation with WoW.

transmissions occur in time slots (transmission opportunities) of fixed length $t_{\text {slot }}$.

The scheduling algorithm we will use here is the GreedyPhysical protocol of Brar et al. [11], up to some changes described in Section V. Our choice is motivated by the fact that GreedyPhysical is based on an accurate physical model of interference that accounts for bi-directional transmissions on a link. Accurate interference modeling has two advantages, i.e.: $i$ ) allowing a higher number of packets to be transmitted in parallel during a time slot with respect to less accurate interference models, such as the protocol model [11], and ii) providing better link quality estimation, thus enabling concurrent scheduling of high quality links only. Moreover, GreedyPhysical retains a sufficiently low computational cost that enables frequent recomputations of the schedule, a key ingredient of our proposed design.

GreedyPhysical takes as input a weighted communication graph, in which a weight $\ell$ on a link represents a demand for $\ell \in \mathbb{N}$ transmission opportunities on that link. The goal of the scheduler is to accommodate all the transmission requests using the minimum possible number of slots, by parallelizing transmissions that do not interfere. However, clients typically do not a priori know the amount of traffic they have to transmit. In fact, in our framework client requirements are expressed in terms of bandwidth (and delay) requirements, which are then converted into aggregate link-level QoS requirements. The key of our design is thus to dynamically take as input these linklevel requirements, to turn them into a set of link weights, and to execute GreedyPhysical in such a way to guarantee the satisfaction of the requirements. The way this is achieved is described in detail in the next subsection.

\section{B. Network operations}

As depicted in Figure 2, transmission opportunities are organized into consecutive periods. Each period $i$ is in turn

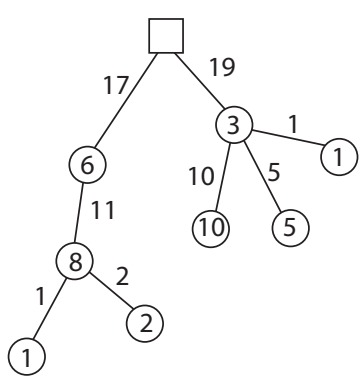

Fig. 3. Example of weight aggregation: nodes are labeled with weights $w_{i}(u)$; links are labeled with the resulting aggregated link weights.

composed of an initial mini-schedule followed by a fixed number $q$ of consecutive identical schedules $S_{i}$.

The schedule $S_{i}$ is computed by the network manager (during period $i-1$ ) as follows. Each node $u$ is first assigned a weight $w_{i}(u)$; node weights (also called node demands) are then aggregated into link weights (see Figure 3) which are given in input to GreedyPhysical. The schedule returned by GreedyPhysical, call it $\hat{S}_{i}$, is then compared against a reference schedule, and the shortest one (in terms of number of allocated time slots) is returned as $S_{i}$. The latter is then broadcast to the gateways, via wired connections, and then to the nodes at the beginning of the $i$-th period (i.e., during the mini-schedule).

The exact choice of the reference schedule depends on the actual bandwidth distribution policy adopted, as will be explained in Section V. However, we must stress here that, if the reference schedule is picked as $S_{i}$, then it must be able to accomodate all the transmission opportunities in $\left\{w_{i}(u)\right\}_{u \in \mathcal{N}}$, where $\mathcal{N}$ is the set of nodes. In the end, $S_{i}$ is a sort of weighted round robin schedule, where some links may even transmit in parallel.

Note that the transmission opportunities allocated in $S_{i}$ to a given node $u$ must be destined by $u$ to both local and transit traffic. More specifically, it is mandated that each node $u$ uses 
exactly $w_{i}(u)$ slots for local traffic, and the remaining slots for transit traffic. Regarding the local traffic, we assume nodes execute a local scheduler, which is in charge of distributing the node's bandwidth among the currently registered clients. The details of the adopted scheduling algorithm, as well as the node-level QoS guarantees it provides, are out of the scope of this paper. In the following, we assume a simple roundrobin or fair-queueing [7], [8], [31] policy is enforced. For what concerns transit traffic, we simply assume that all the traffic that is received/destined from/to descendants in the tree is transmitted in the transit traffic slots. As we shall see, this property is important for providing strong end-to-end delay guarantees to the final user.

The schedule $S_{i}$ is not the only information delivered by the network manager to the nodes in the mini-schedule. Each node $u$ also receives

1. the maximum weight $\hat{w}_{i+1}(u)$ that $u$ will be allowed to require for period $i+1$;

2. the length $\hat{N}_{i+1}$ of the reference schedule that the network manager will use in the next period $(i+1)$, expressed in terms of number of slots. As a consequence of the shortest schedule selection step described above, $\hat{N}_{i+1}$ is also a bound to the length of the next schedule $S_{i+1}$ (i.e., $S_{i+1}$ will last at most $\hat{N}_{i+1} \cdot t_{\text {slot }}$ seconds).

We will refer to the pair $\left\langle\hat{w}_{i+1}(u), \hat{N}_{i+1}\right\rangle$ as to the bandwidth distribution profile of node $u$ for period $i+1$.

Given the guarantee on $S_{i+1}$ 's maximum length, we can define

$$
A B W_{i+1}(u)=\frac{\hat{w}_{i+1}(u)}{\hat{N}_{i+1} t_{\text {slot }}} b_{\text {slot }} \text { bytes } / \text { sec. }
$$

as the link-level available bandwidth guaranteed to node $u$ in period $i+1$, where $b_{\text {slot }}$ is the number of (level 2) bytes transmitted in a slot. The weight aggregation rule used to compute the input to GreedyPhysical (recall Figure 3) ensures that $A B W_{i+1}(u)$ is actually the end-to-end bandwidth from/to node $u$ to/from its gateway. Hence, $A B W_{i+1}(u)$ is the aggregate bandwidth available at node $u$ for accommodating client requests.

This bandwidth is used by the nodes in the following basic admission control (AC) mechanism. Depending on the clients' connection requests and session expirations, node $u$ repeatedly determines the desired bandwidth $B W_{i+1}^{\text {des }}(u)$ for the next period and hence, by inverting formula (1), the desired weight

$$
w_{i+1}^{\text {des }}(u)=\left\lceil B W_{i+1}^{\text {des }}(u) \frac{\hat{N}_{i+1} t_{\text {slot }}}{b_{\text {slot }}}\right\rceil .
$$

Finally, the updated value

$$
w_{i+1}(u)=\max \left\{1, \min \left\{w_{i+1}^{\text {des }}(u), \hat{w}_{i+1}(u)\right\}\right\}
$$

is piggybacked to the network manager in the data traffic at each transmission opportunity. Note that: (1) taking the minimum among $w_{i+1}^{\text {des }}(u)$ and $\hat{w}_{i+1}(u)$ implies that the node might reject or delay some client connection requests; (2) imposing that $w_{i+1}(u) \geq 1$ is required just for piggybacking the information, even in case of no traffic requirements in the current period.
The actual bandwidth guaranteed to node $u$ in period $i+1$ is clearly

$$
B W_{i+1}(u)=\frac{w_{i+1}(u)}{N_{i+1} t_{\text {slot }}} \cdot b_{\text {slot }} \text { bytes } / \text { sec. }
$$

where $N_{i+1}$ may actually be smaller than $\hat{N}_{i+1}$ (which may happen if $w_{i+1}^{\text {des }}(v)<\hat{w}_{i+1}(v)$ at some node $v$ ).

As shown in Section $\mathrm{V}$, the above $\mathrm{AC}$ mechanism and rule for setting the desired weights, combined with proper computation of the maximum allowed weights $\hat{w}_{i+1}$ and the node internal scheduler, ensure that once a client $a$ with QoS demand $\{b w(a), \operatorname{del}(a)\}$ is admitted in the network, its QoS demand is guaranteed to be satisfied during the entire session duration ${ }^{4}$. Also, in Section $\mathrm{V}$ we will see that the node $\mathrm{AC}$ mechanism leads to different AC policies when combined with different network-level bandwidth distribution policies (i.e., with the actual values $\hat{w}_{i+1}(u)$ and $\hat{N}_{i+1}$ delivered by the network manager to the nodes).

At system startup, the network manager sets $w_{0}(u)=$ $\vartheta \in \mathbb{N}, \forall u$. The parameter $\vartheta$ is critical in our design, and it is used to tune the maximum delay vs. bandwidth distribution granularity tradeoff. This tradeoff will be clearly explained in Section V, after bandwidth distribution policies have been introduced. We anticipate that, intuitively speaking, $\vartheta$ determines the duration of the schedule: a higher value of $\vartheta$ results in a longer schedule, which is bad for reducing delay, but allows a finer granularity in the computation of the desired weights, which in turns achieves a better network bandwidth utilization. In practice, $\vartheta$ should be set to the maximum possible value compatible with the application delay requirements. For instance, in the case of VoIP applications, our simulation results show $\vartheta \leq 5$ is feasible.

With regard to delay guarantees, the number $q$ of schedule repeats in each period is set to be at least the maximum height of the trees in the forest. Such a choice, together with the node slot allocation rule (i.e., the fact that, in each schedule $S_{i}$, a node $u$ uses its transmission opportunities in excess of $w_{i}(u)$ for transit traffic) and the weight aggregation rule (recall Figure 3), guarantees that a packet originating at any node reaches its gateway within one period. We stress that, given the accurate interference model used to compute the schedule, hop distance of a node to its gateway is the main factor affecting packet delay.

We conclude this section by observing that, while schedule and bandwidth allocation is computed by the network manager, client connection requests are locally dealt by nodes, i.e., they are managed in a decentralized way. This ensures a considerable signaling reduction with respect to centralized solutions for managing connection requests.

\section{Setting the period length}

Another important parameter in our design is the duration of a period, whose actual value must take conflicting requirements into account. On the one hand, the period length must be long enough for the network manager to compute the schedule

\footnotetext{
${ }^{4}$ This occurs under the assumption that the client does not change AP association.
} 
and bandwidth distribution profiles for the next period. Also, having a long period is good for reducing the impact of the overhead due to the presence of the mini-schedule, which has fixed length. On the other hand, a short period (subject to have $q$ at least as large as the maximum tree heights) is desirable to increase the rate of adaptation of the schedules to the observed traffic.

As far as the (relatively low) computational cost of GreedyPhysical is concerned, it is feasible to think of periods as lasting a few hundred milliseconds for networks of reasonable size (around 100 nodes). In addition, we have verified through simulations that the length of the mini-schedule is actually very short (less that $1 \mathrm{~ms}$ ) for networks of this size. This implies a negligible signaling overhead even when the period length is set to the minimum duration required to execute the network manager algorithms, which is thus the value we have used in our simulations.

\section{BANDWIDTH DISTRIBUTION AND AC POLICIES}

In this section we show two basic bandwidth distribution policies, which stand at opposite ends of the "spatial fairness - network utilization" scale. As we will show, these policies, combined with the AC mechanism described in Section IV-B, give rise to two different $\mathrm{AC}$ policies.

At network startup, both policies compute a first worst-case schedule $S_{0}$, assuming that all the nodes require the maximum possible weight $\vartheta$ (equivalent to continuously backlogged links). Its length is denoted as $N_{0}$. In $S_{0}$ any node $u$ is guaranteed the same bandwidth $B W_{0}(u)=B W_{0}=\frac{\vartheta}{N_{0} t_{\text {slot }}} b_{\text {slot }}$ bytes/sec.

\section{A. Static bandwidth distribution}

The first bandwidth distribution/AC policy, which we call SFC (Spatial Fair, Conservative policy) is a static one. As we already know, at the beginning of each period $i$, the network manager broadcasts, together with the schedule $S_{i}$, also the bandwidth profile $\left\langle\hat{w}_{i+1}(u), \hat{N}_{i+1}\right\rangle$. In SFC, $\hat{w}_{i+1}(u)=\vartheta$ and $\hat{N}_{i+1}=N_{0}$, hence the bandwidth profile is the same for each node $u$ and it is also independent of $i$. SFC guarantees the same bandwidth $B W_{0}$ to each node $u$.

In order to achieve this guarantee, $S_{0}$ is used as the reference schedule in each schedule computation. That is, the schedule $S_{i+1}$ is obtained by comparing the schedule $\hat{S}_{i+1}$, computed by GreedyPhysical, against $S_{0}$ and choosing the shortest one. It is now the appropriate time to explain the need for this additional shortest schedule selection step. The reason is that, given two sets of link weights $W_{1}$ and $W_{2}$, such that $S W_{1} \leq$ $S W_{2}$, where $S W_{i}=\sum_{w \in W_{i}} w, i=1,2$, GreedyPhysical does not guarantee (actually, cannot guarantee) that the schedule for $W_{1}$ is not longer than that for $W_{2}$, as the following example shows.

Consider the weighted communication graph of Figure 4, which also reports the weights $w_{i}(u)$ of each node $u$. Suppose also that, because of interference, the schedule produced by GreedyPhysical is $S_{i}=\langle\{1,3,5\},\{1,3,6\},\{2,4\},\{4,5\}\rangle$, whose length is 4 . Suppose now that $w_{i+1}(u)=w_{i}(u), u \neq 3$, and that node 3 reduces its request at one unit (i.e., $w_{i+1}(3)=$
$1)$. Then it is perfectly conceivable ${ }^{5}$ that interference dependencies impose $S_{i+1}=\langle\{1,3,5\},\{1,4\},\{2,4\},\{5\},\{6\}\rangle$, which is longer than $S_{i}$.

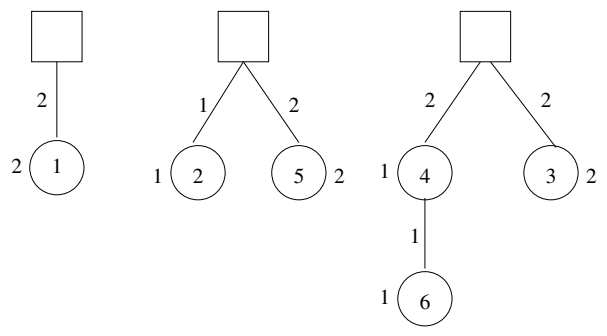

Fig. 4. A communication graph for GreedyPhysical.

Were the actual schedule $S_{i+1}$ longer than $S_{0}$, the bandwidth received by the nodes in the next period would be smaller than $B W_{0}$, and this would lead to a violation of the assumed guarantee. On the contrary, the guarantee is preserved if the shortest schedule between $\hat{S}_{i+1}$ and $S_{0}$ is used. In this respect, we also know that, if the reference schedule $S_{0}$ is picked by the selection step, then it can always accommodate all the transmission opportunities requested by each node $u$, since $w_{i}(u) \leq \vartheta$.

Note, on the contrary, that the actual bandwidth node $u$ receives during the generic period $i$ - see equation (3) - might be higher than $B W_{0}(u)$ : in fact, as already observed, if some of the nodes $v$ is not using all its reserved bandwidth $B W_{0}(v)$, schedule $S_{i}$ is likely to be shorter than $S_{0}$, resulting in a higher bandwidth for some of the nodes. However, our framework guarantees that $B W_{i}$ never goes below $B W_{0}(u)$.

Similarly, the maximum delay for node $u$ is guaranteed to be at most $\operatorname{Del}_{\text {max }}(u)=h(u) \cdot N_{0} \cdot t_{\text {slot }}$, where $h(u)$ is the hopdistance between node $u$ and its gateway. This immediately follows by the weight aggregation rule, and by the fact that nodes use slots in excess to their own weight to transmit transit traffic.

Now, once $B W_{0}(u)=B W_{0}$ and $\operatorname{Del}_{\max }(u)$ are set for each node $u$, the SFC AC policy is straightforward: a new client $a$ requesting a connection with QoS demand $\{b w(a), \operatorname{del}(a)\}$ is admitted if and only if $B W_{0}(u)-$ $B W^{\text {cur }}(u) \geq \operatorname{bw}(a)$ and $\operatorname{del}(a) \geq \operatorname{Del}_{\max }(u)$, where $B W^{\text {cur }}(u) \leq B W_{0}$ is the aggregated bandwidth which is reserved for clients currently registered at node $u$.

The above results can be summarized in the following

Proposition 1: Under the SFC policy, if a client $a$ with QoS demand $\{b w(a), \operatorname{del}(a)\}$ is admitted into the network at node $u$, then $a$ is guaranteed to receive at least $b w(a)$ bandwidth and to incur delay at most $\operatorname{Del}_{\max }(u) \leq \operatorname{del}(a)$.

Note that SFC is spatially fair when distributing bandwidth among nodes. On the other hand, different nodes in the network have different maximum delay guarantees depending on how close they are to their gateways, resulting in spatial unfairness which is not dealt with by SFC. In other words, SFC ensures spatial fairness for what concerns bandwidth, but it is

\footnotetext{
${ }^{5}$ Suppose, for instance, that nodes 5 and 6 are very close to each other.
} 
still not fair for what concerns delay ${ }^{6}$.

It is also worth observing that highly loaded nodes can receive bandwidth in excess to $B W_{0}$. However, owing to SFC's conservative nature, this extra bandwidth can be utilized only for best-effort traffic, and it cannot be used to allocate more clients. In fact, in SFC the aggregate bandwidth guaranteed to a node is computed once and for all at the beginning of the network operation under worst-case assumptions, and it is the same for all the nodes - which ensures spatial fairness in bandwidth allocation.

\section{B. Dynamic bandwidth distribution}

The second bandwidth distribution/AC policy, called SUA (Spatial Unfair, Aggressive policy), sacrifices spatial fairness to improve bandwidth utilization. Differently from SFC, SUA implements dynamic bandwidth allocation to the nodes ${ }^{7}$. The idea is that the extra aggregate bandwidth which is possibly available at the nodes in a certain period can be used to admit more QoS-sensitive clients.

At the beginning of each period $i$, the network manager broadcasts $\hat{w}_{i+1}(u)=w_{i}(u)$ and $\hat{N}_{i+1}=N_{i}$ to each node $u$. The latter, in particular, is a consequence of the fact that, in the SUA policy, we take the current schedule (say, schedule $S_{i}$ ) as the reference schedule for the next period (i.e., period $i+1)$. In other words,

$$
S_{i+1}=\operatorname{argmin}\left\{\operatorname{length}\left(S_{i}\right), \operatorname{length}\left(\hat{S}_{i+1}\right)\right\} .
$$

Similarly to what happens for the static policy, this guarantees that $S_{i+1}$ will not be longer than $N_{i} \cdot t_{\text {slot }}$, and also that the reference schedule can accomodate all node demands, since $w_{i+1}(u) \leq \hat{w}_{i+1}(u)=w_{i}(u)$, for each node $u$.

Each node then enforces the following criterion for admission control (the delay criterion being the same as in SFC): a client $a$ requesting a connection with minimum bandwidth $b w(a)$ is accepted only if $B W_{i}(u)-B W^{c u r}(u) \geq b w(a)$, where $B W_{i}(u)$ is the bandwidth of node $u$ during period $i$ (which, in general, can be different than $B W_{0}$ ). That is, differently from SFC the available aggregate bandwidth is adapted to the current network load.

SUA ensures that nodes can only decrease their weights. As a consequence of this, it has the disadvantage of decreasing the granularity of the bandwidth allocation as time goes by. In the extreme case, all nodes end up with weight 1 , implying that from that point on bandwidth allocation is no longer adaptive to changes in network load. To circumvent this problem, SUA implements the following (weight) push-up rule. Let $w_{i}^{\max }$ be the maximum among node weights during period $i$; then, before invoking GreedyPhysical, all the weights are modified as follows

$$
w_{i+1}(u) \leftarrow w_{i}(u) \cdot\left\lfloor\frac{\vartheta}{w_{i}^{\max }}\right\rfloor
$$

\footnotetext{
${ }^{6}$ Delay unfairness is, in a sense, an intrinsic property of multi-hop wireless communications.

${ }^{7}$ We stress that SUA is actually only one instance of the possible dynamic allocation strategies that can be used in combination with WoW.
}

Proposition 2: Under the SUA policy, if a client $a$ with QoS demand $\{b w(a), \operatorname{del}(a)\}$ is admitted into the network, then $a$ is guaranteed to receive at least $b w(a)$ bandwidth and to incur delay at most $\operatorname{del}(a)$.

To prove it, we first need to prove the following lemma.

Lemma 1: Let $G$ be a communication graph and let $W(G)$ be the set of weights tagging the links of $G$. Also, let $S(W(G))$ denote the schedule produced by Greedy Physical on input the communication graph $G$ with weights $W(G)$. Finally, for integer $t$, let $t \cdot W(G)$ denote the set obtained by multiplying each element of $W(G)$ by $t$. Then $|S(t \cdot W(G))|=$ $t \cdot|S(W(G))|$, where $|S|$ denote the length of schedule $S$.

Proof: We shall prove something stronger, namely that the schedule produced by Greedy Physical on input $t \cdot W(G)$ is obtained by replicating $t$ times each slot in the schedule for $W(G)$.

The proof is by induction on the number of links scheduled. For a graph $G$ with $m$ links, we shall denote with $e_{1}, \ldots, e_{m}$ the links and with $w_{e_{1}}, \ldots, w_{e_{m}}$ the corresponding weights, where the edges are arbitrarily ordered.

The case $m=1$ is trivial, since a link does interfere with itself and thus must be scheduled sequentially. Hence, the schedule produced by Greedy Physical on input the weights $W(G)$ (respectively, $t \cdot W(G)$ ) is exactly $w_{e_{1}}\left(\right.$ resp., $\left.t \cdot w_{e_{1}}\right)$ slots long.

Suppose now, by induction, that the schedule produced by Greedy Physical after examining the first $m-1$ links of $G$ with weights $t \cdot W(G)$ is composed of exactly $t$ replicas of each slot arranged under weights $W(G)$. To help visualize this state of affairs suppose $m=8, W(G)=\langle 1,1,2,1,1,1,2,2\rangle$, $t=3$, and that the schedule produced by Greedy Physical on input $W(G)$ is

$$
\begin{aligned}
S(W(G))= & \left\langle\left\{e_{1}, e_{3}\right\},\left\{e_{2}, e_{8}\right\},\left\{e_{3}, e_{4}\right\},\left\{e_{5}, e_{7}\right\}\right. \\
& \left.\left\{e_{6}\right\},\left\{e_{7}\right\},\left\{e_{8}\right\}\right\rangle
\end{aligned}
$$

Then, the schedule produced by Greedy Physical after step $m-1=7$ on input $3 \cdot W(G)$ is

$$
\begin{aligned}
S(W(G))= & \left\langle\left\{e_{1}, e_{3}\right\},\left\{e_{1}, e_{3}\right\},\left\{e_{1}, e_{3}\right\},\right. \\
& \left\{e_{2}\right\},\left\{e_{2}\right\},\left\{e_{2}\right\}, \\
& \left\{e_{3}, e_{4}\right\},\left\{e_{3}, e_{4}\right\},\left\{e_{3}, e_{4}\right\}, \\
& \left\{e_{5}, e_{7}\right\},\left\{e_{5}, e_{7}\right\},\left\{e_{5}, e_{7}\right\}, \\
& \left\{e_{6}\right\},\left\{e_{6}\right\},\left\{e_{6}\right\} \\
& \left.\left\{e_{7}\right\},\left\{e_{7}\right\},\left\{e_{7}\right\}\right\rangle .
\end{aligned}
$$

To complete the proof, observe that if $e_{m}$ can be scheduled by using $w_{e_{m}}^{\prime} \leq w_{e_{m}}$ new slots in $S(W(G)$, then in $t \cdot W(G)$ it can be scheduled using $t \cdot w_{e_{m}}^{\prime}$ new slots, since (by induction) there are $t \cdot\left(w_{e_{m}}-w_{e_{m}}^{\prime}\right)$ slots in the partial schedule that can accommodate $e_{m}$. For the example above, $w_{e_{m}}^{\prime}=1$ and $t$ out of $2 t$ requests can be accommodated in existing slots of the 
partial schedule:

$$
\begin{aligned}
S(W(G))= & \left\langle\left\{e_{1}, e_{3}\right\},\left\{e_{1}, e_{3}\right\},\left\{e_{1}, e_{3}\right\},\right. \\
& \left\{e_{2} e_{8}\right\},\left\{e_{2}, e_{8}\right\},\left\{e_{2}, e_{8}\right\}, \\
& \left\{e_{3}, e_{4}\right\},\left\{e_{3}, e_{4}\right\},\left\{e_{3}, e_{4}\right\}, \\
& \left\{e_{5}, e_{7}\right\},\left\{e_{5}, e_{7}\right\},\left\{e_{5}, e_{7}\right\}, \\
& \left\{e_{6}\right\},\left\{e_{6}\right\},\left\{e_{6}\right\}, \\
& \left\{e_{7}\right\},\left\{e_{7}\right\},\left\{e_{7}\right\}, \\
& \left.\left\{e_{8}\right\},\left\{e_{8}\right\},\left\{e_{8}\right\}\right\rangle .
\end{aligned}
$$

We can now prove Proposition 2.

Proof: The only difference with respect to the case of SFC is that here we must take possible weight push up into account. Let $G$ be a communication graph and let $W(G)$ be the set of link weights in $G$. Also, for an integer $t$, let $t \cdot W(G)$ denote the set obtained by multiplying each element of $W(G)$ by $t$. Thanks to Lemma 1 the length of the schedule produced by GreedyPhysical on $t \cdot W(G)$ is exactly $t$ times the length of the schedule obtained on input $W(G)$. However, this implies that the in equation (1), both the numerator (i.e., $\hat{w}_{i+1}(u)$ ) and the denominator (i.e., $\hat{N}_{i+1}$ ) are possibly multiplied by the same factor, which clearly implies that the bandwidth guaranteed remains unchanged.

Observe that under the SUA policy it is possible that a node $u$ accepts clients in excess to the fair share $B W_{0}$. This unavoidably compresses the aggregate bandwidth available to some other nodes in the network (which are currently lightly loaded), possibly even below $B W_{0}$. Hence, contrary to SFC, SUA does not guarantee spatial fairness in bandwidth allocation.

\section{Simulations}

In this section we describe our simulation setup and report the obtained results. Simulation results are intended to show that the WoW framework: 1) effectively enables a real-world QoS-sensitive application to run, 2) imposes a negligible overhead and fully utilizes the available total bandwidth, 3) properly distributes this total bandwidth to each node.

We implemented the WoW framework inside the Georgia Tech Network Simulator [2]. More specifically, our starting point was the modified version of the simulator used in [11], which implements the physical interference model and contains the GreedyPhysical scheduler. This version of GTNetS also implements accumulation of interference levels generated by simultaneous transmissions, accounting also for transmissions occurring outside the carrier sensing range of a receiver. Hence, we can consider the results produced by our simulations as relatively accurate. For each scenario, 20 simulation runs of 20 minutes each (simulated time) have been performed. To skip the initial transient period, all average quantities have been computed only for the last 15 minutes of simulation.

Nodes are placed at grid points in a square of side $1400 \mathrm{~m}$. For each simulation run, 10 nodes are randomly selected as gateways, and routing trees are formed as described in Section III. Each node is equipped with a single $54 \mathrm{Mbps}$
Per-node mean clients (alpha $=1$, nodes $=64)$

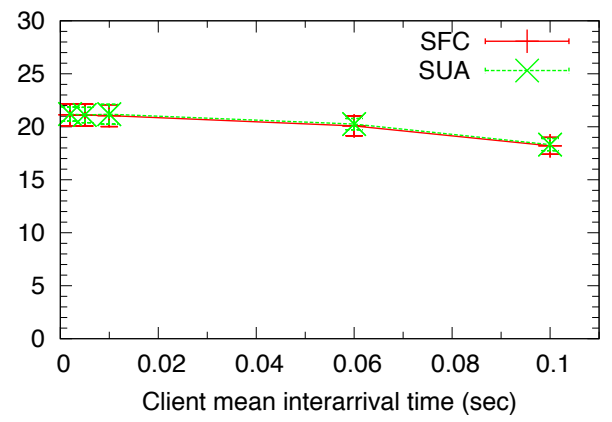

(a)

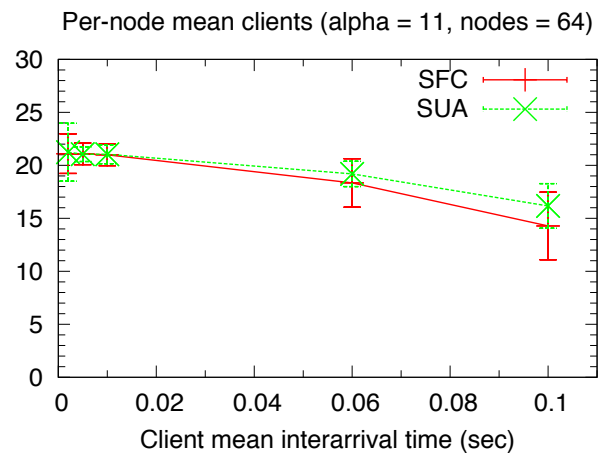

(b)

Fig. 5. Mean number of clients (99\% conf. interval).

bidirectional link. Parameters for the physical interference model are as follows: $200 \mathrm{~mW}$ transmission power, $-90 \mathrm{dBm}$ noise, $22 d B$ SINR threshold. Radio signal propagation obeys log-normal signal propagation, with path-loss exponent 3 and variance $6 d B$. Each slot (transmission opportunity) carries 512 application-level bytes. We used UDP as the transport protocol, which led to a 620 level-2 packet size. Slot duration has been dimensioned to $94 \mu \mathrm{sec}$, namely the minimum possible duration to prevent transmissions in a slot from interfering with the ones of the next slot (i.e., to let the last transmitted bit leave the network before the beginning of the next slot).

With these figures, negligible spatial reuse is possible (the average reuse over all simulations was $0.1 \%$ ). Moreover, each packet reaches the destination gateway in one hop. Such a simple setup has been defined to let the results be clearly due to the WoW framework, without any performance distortion due to unrelated factors such as diversity and spatial reuse. The expected performance with new technologies and/or new underlying schedulers should then be easy to compute. We leave as future work the evaluation of the WoW framework in a more challenging network setting.

As the reference QoS-sensitive application we chose VoIP: arriving clients are randomly associated to a target node, and ask the target node to be admitted to perform a VoIP call. For each simulation run, each node is randomly associated with a popularity index ranging from 1 to 10 . The fact that different APs in a wireless network display different popularity levels is well documented in the literature [5], [6], [19]. Given two nodes $n_{i}$ and $n_{j}$ with popularities $x_{i}$ and $x_{j}, n_{i}$ 's probability 

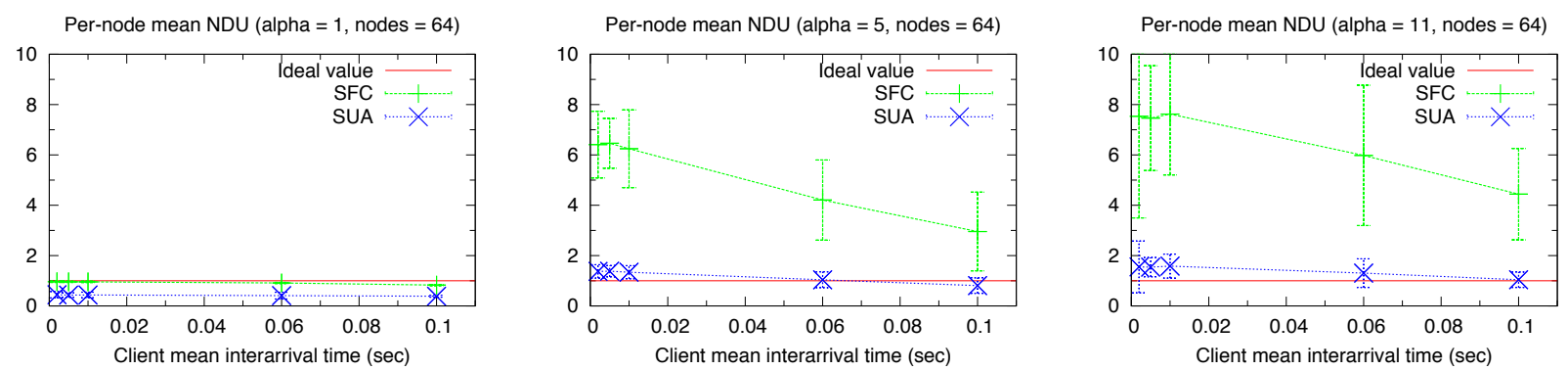

Fig. 6. Mean NDU index (99\% conf. interval) with $\alpha=1$ (left), $\alpha=5$ (center), and $\alpha=11$ ) (right).

to be chosen as target node is $\frac{x_{i}}{x_{j}}$ times higher than the one of $n_{j}$. Node popularity is computed starting from the following truncated power law cumulative distribution $F(x)=x^{\frac{1}{\alpha-1}}$. Here $\alpha$ measures the skewness of the distribution, with $\alpha=1$ meaning that all the nodes have the same popularity. $\alpha$ is a simulation parameter.

Client arrival times follow a Poisson distribution. The mean inter-arrival time $\tau$ is another simulation parameter. Once a client has been admitted, it remains active (i.e., the call lasts) for a random time interval, which is chosen according to an exponential distribution with mean equal to 2 minutes. Policies SFC and SUA are used to perform admission control.

The G.729 Cisco CoDec [1] is used to simulate the VoIP call: each call requires $16 \mathrm{~Kb} / \mathrm{s}$ ( $8 \mathrm{~Kb} / \mathrm{s}$ per audio flow) application-level bandwidth, packets are 78 bytes long and carry a 40 bytes payload. As a simple compression scheme, as many VoIP packets as possible (namely, 6) are encapsulated in each UDP packet. The bandwidth required to accept a new client is computed according to this compression scheme.

Parameter $\vartheta$ in the WoW framework has been set to 5 , and the whole set of different simulated scenarios is given by all the combinations of the following three values: number $n$ of nodes in $\{36,49,64,81,100\}, \alpha \in\{1,3,5,7,9,11\}$, client mean inter-arrival time $\tau \in\{0.01,0.6,0.1\}$ seconds (several extra points have been considered only for the 64 nodes case), SFC and SUA AC policy.

\section{A. Application feasibility}

To evaluate the effectiveness of the framework in implementing the target application, consider that a maximum delay and a maximum jitter of, respectively, $150 \mathrm{~ms}$ and $50 \mathrm{~ms}$ are recommended for a good quality VoIP call (ITU-T G.114 standard). Taking into account all the components of the endto-end delay/jitter in a G.729 packet transmission [1], the mesh network is allowed to introduce a delay/jitter no higher than $35 \mathrm{~ms}$. This constraint is largely fulfilled for network sizes up to 64 nodes, because the maximum observed delay over all simulations for no more than 64 nodes was at most $27 \mathrm{~ms}$. On the contrary $35 \mathrm{~ms}$ and $44 \mathrm{~ms}$ were observed, respectively, for 81 and 100 nodes.

\section{B. Overhead and bandwidth utilization}

The next important figure of merit is the overhead introduced by the framework, which is induced by the presence of
Per-node mean BP $($ alpha $=11$, nodes $=64)$

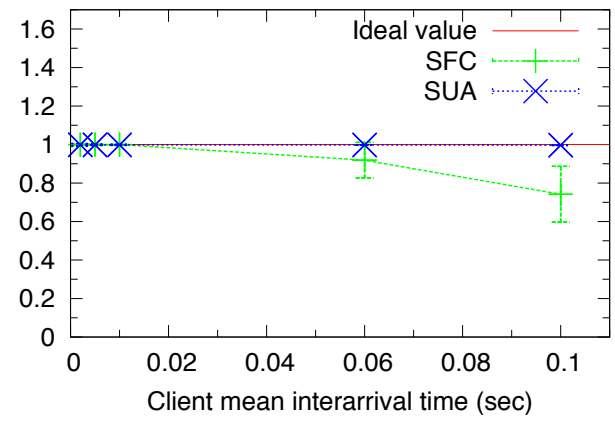

Fig. 7. Mean BP index (99\% conf. interval).

the mini-schedule at each period. The bandwidth distribution profile broadcast to each node was coded on 20 bytes. The resulting maximum observed length of the mini-schedule over all simulations was $155 \mu \mathrm{sec}$. To measure the actual overhead, we can compare the recorded maximum total number of simultaneously active clients against the maximum number achievable in absence of overhead.

An optimistic $3 \%$ reduction of the schedule duration due to spatial reuse, and the hypothesis that each packet be delivered to the destination gateway in one hop would yield a slot rate of 10454 slots/sec. This would allow the transmission of up to 62729 G.729 packets/sec, i.e. the simultaneous service of 1225 clients. Of course, the maximum total number achieved in a simulation run depends both on how high the offered load is, and on how good/bad the client-node association pattern is. I.e., it depends on how frequently a node with no free bandwidth is selected even in presence of not yet saturated ones.

The recorded maximum total number of simultaneously active clients over all simulations is 1200 . It occurred in a run with 64 nodes, $\tau=2 m s, \alpha=11$ and SUA. For all the simulation runs with $\tau \leq 10 \mathrm{~ms}$, both the maximum and the mean total number of clients is higher than 1100. Postponing to the next subsection further investigation on the variance of the number of active clients, we can conclude that the overhead introduced by the framework is practically negligible, and that almost all the available bandwidth is fully utilized. Moreover, $\tau \leq 10 \mathrm{~ms}$ happens to be a saturation load condition for the network. 
Per-node mean NDU (alpha $=11)$

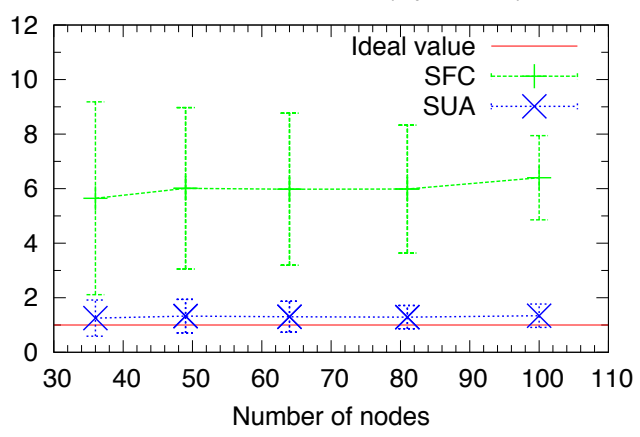

Fig. 8. Mean NDU index (99\% conf. interval) with mean interarrival time equal to $60 \mathrm{~ms}$.

\section{Bandwidth distribution}

Figure 5 shows the per-node mean number of active clients, and the associated $99 \%$ confidence interval, for $\alpha=1$ and $\alpha=11$, respectively. As can be seen, for $\alpha=1$ both policies exhibit low variance around the mean value. In contrast, for $\alpha=11$, the variance dramatically increases as the load gets away from saturation. This is a natural consequence of a highly skewed popularity distribution (intermediate values of the variance would be observed for the other values of $\alpha$ ). The critical issue is whether, in presence of highly skewed popularity distributions, the framework still distributes the available bandwidth to each node proportionally to its popularity.

To investigate this point, for each node and for each period, we computed the following normalized demand utilization (NDU) index: ratio between the node demand during the period and the maximum possible demand (i.e., 5), divided by the ratio between the node popularity and the maximum possible popularity (i.e., 10). Of course, for each node the ideal value of this index should be 1 in stationary, full load condition.

Figures 6 (a), (b) and (c) show the mean value of the index, computed over all the nodes, and the associated $99 \%$ confidence interval, for 64 nodes and $\alpha=1,5,11$. The accuracy of the static policy significantly degrades as $\alpha$ increases. In contrast, SUA achieves a much lower deviation from the ideal value of the index under all values of $\alpha$.

According to equation (2), each node uses the guaranteed maximum length of the schedule for the next period to compute the demand it needs to accommodate the requirements of new clients asking for a connection. Should the resulting demand be higher that the maximum allowed one, these clients would not be admitted. Hence, the performance of both policies in terms of NDU certainly depends both on how close the maximum guaranteed length for the next schedule is to the actual length, and on the maximum allowed node demands.

With regards to the first figure of merit, the bad performance of SFC follows from the fact that, when it comes to admit or not a new client, each node computes the minimum guaranteed bandwidth for the next period as a function of $S_{0}$ length, i.e., as if all the other nodes had maximum demand. Clearly, this may lead to overly pessimistic predictions.

In general, to measure the deviation between the minimum guaranteed bandwidth and the actual one, we have computed the following bandwidth prediction (BP) index for a given node in a given period: ratio between the node guaranteed bandwidth for the next period (according to the guaranteed maximum schedule length), and the actual node bandwidth recorded in the next period. Figure 7 shows the mean value of the index, computed over all the nodes, and the associated $99 \%$ confidence interval, for 64 nodes and $\alpha=11$. Also for the other values of $\alpha$ (plots not reported for brevity), SUA policy is very close to optimum, whereas SFC performance significantly degrades as $\alpha$ increases.

Nevertheless, even if at a considerable lower extent, the SUA policy deviates from the ideal distribution as well, especially for $\alpha=1$ and $\alpha=11$ (Figure 6). This deviation is related to the second figure of merit, i.e., the maximum allowed demands for the nodes. In this respect, SUA forcibly caps the next possible maximum demand of a node to its value for the current period. This can cause the maximum allowed demand for some nodes to decrease as time passes. A higher maximum demand should be recovered by periodically pushing up all the weights according to equation (4). However, from Figure 6 (a) it can be deduced that the mean node demand does end up being quite low. This coarsens the granularity of the possible node demand values, and hence degrades the accuracy in distributing the bandwidth to nodes with different popularities, as it can be seen from figures 6 (b) and (c). Basically, due to the coarse demand granularity, low popularity nodes unfairly steal more bandwidth than needed.

Finally, to show the accuracy of the framework in distributing the network bandwidth for the different network sizes, the NDU index for $\alpha=11$ and $\tau=60 \mathrm{~ms}$ (the configuration for which both SUA and SFC showed the worst NDU values) is reported as a function of the number of nodes in Figure 8.

\section{CONCLUSIONS AND FUTURE WORK}

In this paper, we have introduced the WoW framework for providing strong QoS guarantees in wireless mesh networks. Differently from most proposals for wireless mesh networks, our framework is based on Spatial-TDMA coupled with a very accurate interference model. With respect to CSMA-based approaches, our approach has the advantage of enabling accurate end-to-end bandwidth/delay estimation with a negligible signaling overhead.

To complement the proposed framework, we have proposed two simple backbone node-level bandwidth distribution/AC policies that stand at opposite ends of the spatial fairness/bandwidth utilization tradeoff. Through extensive simulations with accurate interference modeling, we have verified WoW's effectiveness in providing strong QoS guarantees to VoIP users in a realistic mesh network scenario. Furthermore, we have shown WoW's ability to provide these guarantees and to effectively use network bandwidth even in presence of highly unbalanced network load. To the best of our knowledge, WoW is the first framework for QoS provisioning in mesh networks with similar features proposed in the literature.

The work presented in this paper leaves several issues open for further research. First, more sophisticated backbone 
node-level policies can be implemented. In particular, policies for dynamic bandwidth allocation allowing a better weight management than SUA are needed. Most importantly, our framework needs to be extended to include more flexible traffic and client mobility patterns. For what concerns traffic, our framework currently assumes that all communications are from/to nodes to/from gateways, while node-to-node communications might in general occur for at least some wireless mesh network deployment scenarios (e.g., community networking). Implementing node-to-node communications might require integrating our framework with a more sophisticated, QoS-aware, routing approach. For what concerns client mobility, our framework currently delivers strong QoS guarantees only under the assumption that a client does not change AP association during a session. In presence of client mobility, QoS-aware handoff strategies should be implemented, along the lines, e.g., of those presented in [37]. In this context, the tradeoff between leaving some unused bandwidth at each node to account for roaming clients and overall network bandwidth utilization is to be carefully evaluated. In this respect, client mobility and behavioral models such as those proposed, e.g., in [21], [23] might prove useful in forecasting a client's next AP association, thus enabling a more accurate QoS provisioning.

\section{REFERENCES}

[1] http://www.cisco.com/en/US/tech/tk652/tk698/technologies_white_paper09186a00800a8993.shtml\#buildingdelaybudget

[2] http://www.ece.gatech.edu/research/labs/MANIACS/GTNetS/

[3] M. Alicherry, R. Bathia, L. Li, "Joint Channel Assignment and Routing for Throughput Optimization in Multi-Radio Wireless Mesh Networks", Proc. ACM Mobicom, pp. 58-72, 2005.

[4] B. Aoun, R. Boutaba, Y. Iraqi, G. Kenward, "Gateway Placement Optimization in Wireless Mesh Networks with QoS Constraints", IEEE JSAC, to appear.

[5] A. Balachandran, G.M. Voelker, P. Bahl, P. Venkat Rangan, "Characterizing User Behavior and Network Performance in a Public Wireless LAN", Proc. ACM Sigmetrics 02, pp. 195-205, 2002.

[6] M. Balazinska, P. Castro, "Characterizing Mobility and Network Usage in a Corporate Wireless Local-Area Network", Proc. ACM MobiSys 03, pp. 303-316, 2003.

[7] J.C.R. Bennett, H. Zhang, "WF' Q: Worst-Case Fair Weighted Fair Queueing", Proc. INFOCOM, pages 120-128, 1996.

[8] J.C.R. Bennett, H. Zhang, "Hierarchical packet fair queueing algorithms", IEEE/ACM Transactions on Networking, pages 675-689, 1997.

[9] N. Ben Salem and J.P. Hubaux, "A Fair Scheduling for Wireless Mesh Networks", Proc. IEEE WiMesh, 2005.

[10] Y. Bejerano, "Efficient Integration of Multi-hop Wireless and Wired Networks with QoS Constraints", IEEE/ACM Trans. on Networking, Vol. 12, n. 6, pp. 1064-1078, 2004.

[11] G. Brar, D. Blough, and P. Santi, "Computationally Efficient Scheduling with the Physical Interference Model for Throughput Improvement in Wireless Mesh Networks," Proc. ACM Mobicom, pp. 2-13, 2006.

[12] G.C. Clark Jr., C.J. Bibb, "Error-Correction Coding for Digital Communications", New York: Plenum Press, 1981.

[13] X. Chu, "Provisioning of Parametrized Quality of Service in 802.11e Based Wireless Mesh Networks", Mobile Networks and Applications, Vol. 13, pp. 6-18, April 2008.
[14] D. Dharmaraju, A.R. Chowdhury, P. Hovareshti, J.S. Baras, "INORA: A Unified Signalling and Routing Mechanism for QoS Support in Mobile Ad Hoc Networks", Proc. ICPPW 2002, pp. 86-93, 2002.

[15] V. Gambiroza, B. Sadeghi, E.W. Knightly, "End-to-End Performance and Fairness in MultiHop Wireless Backhaul Networks", Proc. ACM Mobicom, pp. 287-301, 2004.

[16] P. Gupta and P.R. Kumar, "The Capacity of Wireless Networks," IEEE Transactions on Information Theory, Vol. 46, No. 2, pp. 388-404, 2000.

[17] M. Kodialam, T. Nandagopal, "Characterizing the Capacity Region in Multi-Radio Multi-Channel Wireless Mesh Networks", Proc. ACM Mobicom, pp. 73-87, 2005.

[18] V. Kone, S. Das, B.Y. Zhao, H. Zheng, "QUORUM: QUality Of service RoUting in wireless Mesh networks", Mobile Networks and Applications, Vol. 12, pp. 358-369, Dec. 2007.

[19] D. Kotz, K. Essien, "Characterizing Usage of a Campus-Wide Wireless Network", Proc. ACM Mobicom, pp. 107-118, 2002.

[20] P. Lassila, A. Penttinen, J. Virtamo, "Dimensioning Wireless Mesh Networks with Flow-Level QoS Requirements", Proc. ACM PE-WASUN, pp. 17-24, 2006.

[21] J.K. Lee, J. Hou, "Modeling Steady-State and Transient Behaviors of User Mobility: Formulation, Analysis, and Application", Proc. ACM MobiHoc 06, pp. 85-96, 2006.

[22] S.B. Lee, A. Gahng-Seop, X. Zhang, A.T. Campbell, "INSIGNIA: An IP-based Quality of Service Framework for Mobile Ad Hoc Networks", Journal of Parallel and Distributed Computing, Vol. 60, n.4, pp. 374-406, 2000.

[23] D. Lelescu, U. Kozat, R. Jain, M. Balakrishnan, "Model T++: An Empirical Joint Space-Time Registration Model", Proc. ACM MobiHoc 06, pp. 61-72, 2006.

[24] C.R. Lin, J. Liu, "QoS Routing in Ad Hoc Wireless Networks", IEEE JSAC, Vol. 17, n. 8, pp. 1426?1438, 1999.

[25] C.R. Lin, "On-demand QoS routing in Multihop Mobile Networks", Proc. IEEE Infocom, pp. 1735-1744, 2001.

[26] L. Luo, M. Gruteser, H. Liu, K. Huang, S. Chen, "A QoS Routing and Admission Control Scheme for 802.11 Ad Hoc Networks", Proc. ACM Diwans, pp. 19-27, 2006.

[27] R. Maheshwari, S. Jain, S.R. Das, "A Measurement Study of Interference Modeling and Scheduling in Low-Power Wireless Networks", Proc. 6th ACM Conference on Embedded Networked Sensor Systems (ACM SenSys 2008), Raleigh, NC, Nov 2008.

[28] R. Maheshwari, J. Cao, S.R. Das, "Physical Interference Modeling for Transmission Scheduling on Commodity WiFi Hardware", submitted for publication, 2008.

[29] S. Mangold, S. Choi, P. May, O. Klein, G. Hiertz, L. Stibor, "IEEE 802.11e Wireless LAN for Quality of Service", Proc. European Wireless, pp. 32-39, 2002.

[30] R. Nelson and L. Kleinrock, "Spatial-TDMA: A Collisonfree Multihop Channel Access Protocol," IEEE Transactions on Communication, Vol. 33, pp. 934-944, Sept. 1985.

[31] A.K. Parekh, R.G. Gallager, "A generalized processor sharing approach to flow control in integrated services networks-the single node case", Proc. IEEE INFOCOM '92, 1992, pages 915924, Florence, Italy

[32] B. Raman, K. Chebrolu, "Design and Evaluation of a new MAC Protocol for Long-Distance 802.11 Mesh Networks" Proc. ACM Mobicom, pp. 156-169, 2005.

[33] T.B. Reddy, I. Karthigeyan, B.S. Manoj, C. Siva Ram Murthy, "Quality of Service Provisioning in Ad Hoc Wireless Networks: a Survey of Issues and Solutions", Ad Hoc Networks, Vol. 4, n. 1, pp. 83-124, 2006.

[34] S. Sheu, T. Sheu, "DBASE: A Distributed Bandwidth Allocation/Sharing/Extension Protocol for Multimedia over IEEE 802.11 Ad Hoc Wireless LAN", Proc. IEEE Infocom, pp. 15581567, 2001.

[35] P. Sinha, R. Sivakumar, V. Bharghavan, "CEDAR: A Core Extraction Distributed Ad Hoc Routing Algorithm", IEEE JSAC, Vol. 17, n. 8, pp. 1454-1466, 1999. 
[36] S. Sriram, T.B. Reddy, B.S. Manoj, C. Siva Ram Murthy, "On the End-to-end Call Acceptance and the Possibility of Deterministic QoS Guarantees in Ad Hoc Wireless Networks", Proc. ACM MobiHoc, pp. 169-180, 2005.

[37] Y. Wei, R. Maosheng, T. Zhao, L. Xiaoming, "A Bandwidth Management Scheme Support for Real-time Applications in Wireless Mesh Networks", Proc. ACM SAC, pp. 2063-2068, 2008.

[38] R. Zhao, B. Walke, "Decentrally Controlled Wireless MultiHop Mesh Networks for High Quality Multi-media Communications", Proc. ACM MSWiM, pp. 200-208, 2005. 